Send your research letters to the editor, British Dental Journal, 64 Wimpole Street, LondonW1G8YSE-mail bdj@bda.org Priority will be given to letters less than 500 words long. Authors must sign the letter, which may be edited for reasons of space

\section{Laser legislation}

Sir, The Healthcare Commission has been concerned for some time about anomalies regarding the regulation of dentists in England who use a Class 4 laser or an Intense Pulsed Light source within their practice. The Healthcare Commission says that dentists have been included under regulation within the Care Standards Act 2000 (CSA) and dentists are affected by the legislation in respect of both NHS and private practices. However, this has been an issue of some contention, as some dentists did not consider that the new legislation applies to their service and there was a lack of clarity in relation to whether dentists were required to be registered with the National Care Standards Commission (NCSC).

The Healthcare Commission is now in receipt of definitive legal advice that advises that dental practitioners carrying out, or wishing to carry out, Class 4 laser treatment must be registered under the Care Standards Act 2000 (CSA), irrespective of whether their lists are wholly private or NHS or a combination of both.

A joint consultation meeting between the Healthcare Commission, the General Dental Council, the British Dental Association and the General Dental Practitioners' Association took place last year to discuss the way forward. The GDPA has expressed concern about both the cost of inspection and the further administrative burden placed on dental practices by this scheme. Inspections may take up to three hours, however much of the information required should already be readily available for routine PCT practice visits under NHS contracts, vocational training inspections or BDA Good Practice assessment.

The Healthcare Commission wants to foster a collaborative approach towards bringing dentists who use Class 4 lasers or Intense Pulsed Light treatments into regulation under the CSA and is advising dentists to apply for registration or they will be considered to be running an unregistered service, which is an offence under the Care Standards Act 2000. Annual inspections are expected to cost at least $£ 1,500$ and as there is little likelihood that this fee will be supported by PCTs, the cost will probably contribute to the price of private laser treatment borne by patients.

The Healthcare Commission will endeavour to assist dentists as much as possible in understanding the regulatory process as it affects dental practices and to answer any queries dentists may have. If dentists use class 4 lasers and are not registered under the CSA regulations they are urged to contact the Healthcare Commission at Finsbury Tower, 103-105 Bunhill Row, London EC1Y 8TG or telephone them on 02074489200 as soon as possible.

\section{N. Entwistle}

Dental Adviser - The Healthcare Commission By email doi: 10.1038/sj.bdj.4812662

\section{Studies in osteonecrosis}

Sir, it was with great interest that I read the recent letter published in the $B D J$ Special precautions (BDJ 2005; 198: 628).

We have been looking into this subject. There have been various studies carried out on the effects of Zometa and Aredia on bony metastasis from tumours such as breast cancer and prostate cancer. It has been found to reduce the overall risk of developing bone complications and also helps reduce bone pain. The expert panel of the American Society of Clinical Oncology state that bisphophonates reduce bone complications but do not, however, have an impact on patient survival, ie they improve the quality of life rather than cure the disease.

In the last few years oncologists/ surgeons have become aware of an increase in the number of patients with osteonecrosis, in patients taking either Zometa or Aredia.

Osteonecrosis is a rare and poorly understood condition that is often

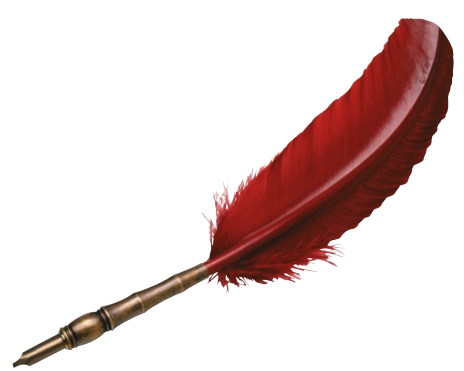

mistaken for other conditions of the jaw such as osteomyelitis, exposed bone, necrosis and impaired healing after a dental procedure. The symptoms of osteonecrosis include pain, swelling, gum infections, loosening of teeth, poor healing of gums - especially after dental treatment - and numbness or heavy feeling in the jaw. Treatment for osteonecrosis includes antibiotics, oral rinses and protective mouth guards.

Osteonecrosis and its complications can lead to chronic pain, dysfunction and disfigurement all of which may be difficult to treat.

There have been various papers published about the association of Zometa, Aredia and osteonecrosis. A case report published in the Journal of Oral Pathology and Medicine in February 2005 (J Oral Pathol Med 2004; 33: 1-4) looked at 10 patients with osteonecrosis of the jaw that appeared following cancer chemotherapy. Six of the patients had bone metastases from breast cancer and four had multiple myelomas. All 10 patients had osteonecrosis of the mandible; 50\% also had maxillary involvement. In all 10 patients the histopathological diagnosis was of chronic osteomyelitis without evidence of metastatic disease of the bone. Seven of the patients had a tooth extraction prior to the onset of the osteonecrosis. All the patients had received Zometa and/or Areida as part of their treatment. The authors conclude that osteonecrosis appears to have a relationship with the use of the bisphosphonates Zometa and Aredia.

More research is needed, as these patients are usually on other treatments such as chemotherapy or radiotherapy that may contribute to the development of osteonecrosis.
S. Makani
C. Mander
M. Cox
K. Wragg
Eastern Leicester and Erewash PCTs
By email
doi: $10.1038 /$ sj.bdj.4812663 